\title{
MEAN FIELD ANALYSIS OF TWO COUPLED HEISENBERG MODELS
}

\author{
D. V. Shopova ${ }^{1}$, T. L. Boyadjiev ${ }^{2}$ \\ ${ }^{1}$ CPCM Laboratory, G. Nadjakov Institute of Solid State Physics \\ Bulgarian Academy of Sciences, BG-1784 Sofia, Bulgaria \\ ${ }^{2}$ Faculty of Mathematics and Computer Science, University of Sofia \\ $B G-1164$ Sofia, Bulgaria \\ (Received October 2, 2001)
}

\begin{abstract}
We have derived and analyzed the Landau free energy for two coupled Heisenberg subsystems in the general case of $n$-component order parameters. The calculation was done in the weak-coupling limit when the exchange interactions $J_{1}$ and $J_{2}$ in the subsystems exceed the intersubsystem interaction $K$. There exist two stable collinear phases, depending on the sign of $K$. The phase transitions to the stable phases are of second order for the whole range of parameters of the model under consideration. The numerical calculations show that for positive sign of interactions two more phases exist, which are metastable or unstable depending on the number of the order parameter components $n$.

Key words: Heisenberg model, magnetism, order parameter, phase transition.
\end{abstract}

PACS number(s): 75.10.Hk,75.30.Kz,75.40.Cx

\section{INTRODUCTION}

The model of two bilinearly coupled Heisenberg subsystems composed of $n$-component classical spins is interesting for the understanding of the magnetic properties of substances with complex magnetic structure. Generally, this model can be applied to two classes of magnetic materials. The first class comprises substances with one type of magnetic ions which occupy crystallographically nonequivalent positions in the Bravais lattice and are surrounded by different number or type of nonmagnetic ions. Substances which contain two chemically different types of magnetic ions fall in the second class; here the superlattices composed of two different magnetic materials can be included, too. The resulting effect is that the inital system can be considered as two coupled subsystems having different effective exchange interactions no matter of the microscopic origin of this difference.

The model, of course, is quite general and can be used for the analysis of the properties also in other systems. An example for its application to the granular superconductors is given in book [1]. In this paper we shall consider the application of the model only to magnetic materials. For this purpose we shall derive the general Ginzburg-Landau free energy functional from the respective microscopic model and establish the relation between the interaction parameters of the microscopic Hamiltonian and the macroscopic Landau parameters. We shall analyze numerically the obtained mean-field equations with the aim to identify the ordered phases and investigate their stability.

\section{DERIVATION OF LANDAU FREE ENERGY}

To do the mean-field analysis and describe the properties of magnetically ordered phases we should obtain an expression for the Landau free energy and the connection of its coefficients with the microscopic parameters. We start the derivation with the following microscopic Hamiltonian:

$$
\begin{aligned}
H & =-\frac{1}{2} \sum_{i j}^{2 N}\left[J_{i j}^{(1)} \mathbf{S}_{i}^{(1)} \cdot \mathbf{S}_{j}^{(1)}+J_{i j}^{(2)} \mathbf{S}_{i}^{(2)} \cdot \mathbf{S}_{j}^{(2)}\right. \\
& \left.+2 K_{i j} \mathbf{S}_{i}^{(1)} \cdot \mathbf{S}_{j}^{(2)}\right],
\end{aligned}
$$

which describes two bilinearly coupled classical Heisenberg models. Here $\mathbf{S}_{i}^{\alpha}$ are $n$-component classical spin vectors which are normalized by the condition $\left|\mathbf{S}_{i}^{(\alpha)}\right|=1$; $(\alpha=1,2)$. The dimensionless exchange parametres,

$$
\begin{gathered}
J_{i j}^{(\alpha)}=\frac{\mathcal{J}_{i j}^{(\alpha)}}{T}, \\
K_{i j}=\frac{\mathcal{K}_{i j}}{T},
\end{gathered}
$$

denote the interaction in the subsystems $\alpha,(\alpha=1,2)$ and between them, respectively, and in the general case are the elements of $(N \times N)$ symmetric matrices. We suppose from the very beginning that the number of lattice sites of both subsystems is one and the same. This is a simplifing condition because it makes the system fully symmetric with respect to the interchange of its parts, i. e., $1 \leftrightarrow 2$.

In order to find the mean field energy we have to calculate the partition function which for the Hamiltonian (1) is represented by an integral over $n$-dimensional unit sphere. We can avoid the integration in the nonEucledian spin space by passing to real space vector fields through the Hubbard-Stratonovich transformation 


\section{V. SHOPOVA, T. L. BOYADJIEV}

(HST) (a systematic presentation of HST is given in Refs. $[2,3]$ ). There are different ways to apply this transformation for complex models; see, for example [1,4]. For our aims it will be more adequate to use the approach of Mukadam and Uzunov [4] which in the original paper is given for two coupled Ising models but can be generalized for the $n$-component vector model, too. The main advantages in their derivation are the direct connection of the order parameters with physical quantities like, for example, the sublattice magnetizations and the fact that the symmetry of the initial model is explicitly preserved in the final result. This is very important for the proper identification of the ordered magnetic phases.

We shall not give details in the application of HST, rather we shall write down the obtained effective Hamiltonian:

$$
\begin{aligned}
\mathcal{H} & =\frac{1}{2} \sum_{i j}^{2 N}\left\{J_{i j}^{(1)} \boldsymbol{\Phi}_{i} \cdot \boldsymbol{\Phi}_{j}+J_{i j}^{(2)} \boldsymbol{\Psi}_{i} \cdot \boldsymbol{\Psi}_{j}+2 K_{i j} \boldsymbol{\Phi}_{i} \cdot \boldsymbol{\Psi}_{j}\right\} \\
& -\ln \sum_{i}^{2 N}\left\{I_{n / 2-1}\left|\sum_{j}^{2 N}\left(J_{i j}^{(1)} \boldsymbol{\Phi}_{j}+K_{i j} \boldsymbol{\Psi}_{j}\right)\right|\left[\left|\sum_{j}^{2 N}\left(J_{i j}^{(1)} \boldsymbol{\Phi}_{j}+K_{i j} \mathbf{\Psi}_{j}\right)\right| / 2\right]^{-n / 2} \Gamma\left(\frac{n}{2}\right)\right\} \\
& -\ln \sum_{i}^{2 N}\left\{I_{n / 2-1}\left|\sum_{j}^{2 N}\left(J_{i j}^{(2)} \boldsymbol{\Psi}_{j}+K_{i j} \boldsymbol{\Phi}_{j}\right)\right|\left[\left|\sum_{j}^{2 N}\left(J_{i j}^{(2)} \mathbf{\Psi}_{j}+K_{i j} \boldsymbol{\Phi}_{j}\right)\right| / 2\right]^{-n / 2} \Gamma\left(\frac{n}{2}\right)\right\} .
\end{aligned}
$$

Here $I_{n / 2-1}\left(x_{i}^{(1,2)}\right)$ is the modified Bessel function and $\Gamma(n / 2)$ is the Gamma function; $\boldsymbol{\Phi}_{i}$ and $\boldsymbol{\Psi}_{i}$ are $n$ component real vector fields which correspond to $\mathbf{S}_{i}^{(1)}$ and $\mathbf{S}_{i}^{(2)}$, respectively. The dimensionless Landau energy per particle, $g=G / N T$, can be obtained from the above expression by expanding it for small values of $|\boldsymbol{\Phi}|$ and $|\boldsymbol{\Psi}|$. Note, that this means variables

$$
x_{i}^{(1)}=\left|\sum_{j}^{2 N}\left(J_{i j}^{(1)} \boldsymbol{\Phi}_{j}+K_{i j} \mathbf{\Psi}_{j}\right)\right|
$$

and

$$
x_{i}^{(2)}=\left|\sum_{j}^{2 N}\left(J_{i j}^{(2)} \mathbf{\Psi}_{j}+K_{i j} \boldsymbol{\Phi}_{j}\right)\right|
$$

to be less than unity.

To be more concrete we shall do the calculations for a body-centered cubic lattice, which contains two different magnetic ions in the elementary cell. In our investigation we neglect finite size and surface effects, that is why we can pass in a standard way to the continium limit in k-space. The Fourier transformed expression of Eq. (3) again contains a bilinear term and we have to diagonalize its quadratic part. This is done with the help of the unitary matrix $\hat{S}$ :

$$
\hat{S}=\frac{1}{D_{s}(\mathbf{k})}\left(\begin{array}{cc}
S_{0}(\mathbf{k}) & -S_{1}(\mathbf{k}) \\
S_{1}^{*}(\mathbf{k}) & S_{0}(\mathbf{k})
\end{array}\right) .
$$

The matrix elements are given by the following expressions and are $\mathbf{k}$-dependent:

$$
\begin{aligned}
& S_{0}(\mathbf{k})=J_{1}(\mathbf{k})-J_{2}(\mathbf{k})+\sqrt{\left[J_{1}(\mathbf{k})-J_{2}(\mathbf{k})\right]^{2}+4 K^{2}(\mathbf{k})}, \\
& S_{1}(\mathbf{k})=2 K(\mathbf{k}) .
\end{aligned}
$$

The function

$$
D_{s}(\mathbf{k})=\sqrt{2}\left\{\left[J_{1}(\mathbf{k})-J_{2}(\mathbf{k})\right]^{2}+4 K^{2}(\mathbf{k})\right\}^{1 / 4}
$$

$$
\times\left\{\sqrt{\left[J_{1}(\mathbf{k})-J_{2}(\mathbf{k})\right]^{2}+4 K^{2}(\mathbf{k})}+J_{1}(\mathbf{k})-J_{2}(\mathbf{k})\right\}^{1 / 2}
$$

ensures that Det $\hat{S}=1$.

Following the standard mean-field procedure we have to find the eigenvalues of the matrix $\hat{S}$ :

$$
\begin{aligned}
\lambda_{1,2}(\mathbf{k}) & =\frac{1}{2}\left[J_{1}(\mathbf{k})+J_{2}(\mathbf{k})\right. \\
& \left. \pm \sqrt{\left[J_{1}(\mathbf{k})-J_{2}(\mathbf{k})\right]^{2}+4 K^{2}(\mathbf{k})}\right]
\end{aligned}
$$

They enter in the coefficients of the diagonalized quadratic part of the effective Hamiltonian in the following way: 


$$
\mathcal{H}^{(2)} \sim\left(1-\frac{\lambda_{1}(\mathbf{k})}{n}\right) \boldsymbol{\varphi}_{1}^{2}(\mathbf{k})+\left(1-\frac{\lambda_{2}(\mathbf{k})}{n}\right) \boldsymbol{\varphi}_{2}^{2}(\mathbf{k}) .
$$

It is obvious that there may be vectors $\mathbf{k}$, for which $\lambda_{1,2}(\mathbf{k})$ will be negative. The procedure which excludes such values of $\mathbf{k}$ is well described in Ref. [1]. Practically we have to find those values $\mathbf{k}_{0}$ which give maxima of $\lambda_{1,2}(\mathbf{k})$. Mathematically this is equivalent to the evaluation of the integral for the partition function by the saddle point method.

For the bcc lattice in the nearest-neighbour approximation these maxima can be found analytically in two limiting cases. We are interested only in the so-called weak coupling limit when the exchange interaction in the subsystems is bigger than the subsystem coupling. In this case there are two possibilities: (1) if the in-subsystem exchanges are positive, the maxima of eigenvalues are reached for $\mathbf{k}=0$, and (2) if in-subsystem exchanges are negative the maxima of $\lambda_{1,2}(\mathbf{k})$ will be for $|\mathbf{k}|=\pi / a$, where $a$ is the lattice constant.

We shall consider in details only a positive exchange in the subsystems. Then the initial system can be presented as two interpenetrating ferromagnetically ordered sublattices which interact either ferro- or antiferromagnetically depending on the sign of intersublattice exchange. To put all these conclusions in a mathematical form we introduce the following notations:

$$
\begin{aligned}
& J_{1,2}=J_{1,2}(\mathbf{k}=0) ; \\
& K=K(\mathbf{k}=0) ; \\
& \lambda_{1,2}=\lambda_{1,2}(\mathbf{k}=0) .
\end{aligned}
$$

The next steps include an expansion of $J_{1,2}(\mathbf{k})$, and $K(\mathbf{k})$ in the vicinity of $\mathbf{k}=0$ and the inverse Fourier transform of the obtained expression to the real space. As a result a Ginzburg-Landau functional is obtained. We shall not study fluctuations and shall take only the space independent part of it:

$$
\begin{aligned}
g & \equiv \frac{G}{N T}=\frac{\tau_{1}}{2} \boldsymbol{\varphi}_{1}^{2}+\frac{\tau_{2}}{2} \boldsymbol{\varphi}_{2}^{2}+\frac{g_{1}}{4}\left(\boldsymbol{\varphi}_{1}^{2}\right)^{2} \\
& +\frac{g_{2}}{4}\left(\boldsymbol{\varphi}_{2}^{2}\right)^{2}+\frac{v}{2} \boldsymbol{\varphi}_{1}^{2} \boldsymbol{\varphi}_{2}^{2}+v\left(\boldsymbol{\varphi}_{1} \cdot \boldsymbol{\varphi}_{2}\right)^{2} \\
& +w_{1} \boldsymbol{\varphi}_{1}^{2}\left(\boldsymbol{\varphi}_{1} \cdot \boldsymbol{\varphi}_{2}\right)+w_{2} \boldsymbol{\varphi}_{2}^{2}\left(\boldsymbol{\varphi}_{1} \cdot \boldsymbol{\varphi}_{2}\right) .
\end{aligned}
$$

This is the expression for the mean-field Landau free energy per particle in dimensionless units, see also Eq. (2) for the model (1).

The parameters of the Landau energy (9) are given in an explicit form with the help of the averaged over nearest neighbours initial microscopic exchange parameters:

$$
\tau_{1,2}=1-\frac{\lambda_{1,2}}{n},
$$

$$
\begin{aligned}
g_{1,2} & =\frac{u}{2} \lambda_{1,2}^{2}\left(S_{1}^{4}+S_{0}^{4}\right), \\
v & =u \lambda_{1} \lambda_{2} S_{1}^{2} S_{0}^{2}, \\
w_{1,2} & =\mp \frac{u}{2} \lambda_{1,2} \sqrt{\lambda_{1} \lambda_{2}} S_{0} S_{1}\left(S_{0}^{2}-S_{1}^{2}\right) .
\end{aligned}
$$

Here $S_{1,0}=S_{1,0}(\mathbf{k}=0)$, see Eqs. (5). The number of the order parameter components enter in Eqs. (10) through the quantity:

$$
u=n^{2}(n+2) \text {. }
$$

For equivalent sublattices, i. e., $J_{1}=J_{2}=J^{0}, w_{1,2}^{0} \equiv$ 0 , and $S_{1}^{0} \equiv S_{0}^{0}=1 / \sqrt{2}$, and the expression for the Landau energy becomes quite simple and can be considered analytically. We should mention that the above expression for the Landau energy is valid not only for the weak coupling limit $\left(J_{1} J_{2}-K^{2}\right)>0$ but also for an arbitrary value of the relation $\left(J_{1} J_{2}-K^{2}\right)$ between the exchange parameters $J_{1}, J_{2}$ and $K$.

The same form of Landau energy was derived with the help of symmetry group considerations by Oleksy and Przystawa [5]. They claim that the two bilinearly coupled order parameters are transformed under two equivalent irreducible representations, i. e., the resulting energy is obtained through a reducible representation and nevertheless the phase transitions are of second order. The last statement violates the Landau condition for the appearance of second order phase transition, namely, the requirement of the irreducibility of representation. As far as there are no details of the derivation in the original paper we can hardly make a connection between our results and their conclusions.

\section{MEAN-FIELD EQUATIONS}

The mean-field equations are obtained as usual by the condition

$$
\frac{\partial g}{\partial \varphi_{1 i}}=0, \quad \frac{\partial g}{\partial \varphi_{2 i}}=0, \quad(i=1, \ldots, n),
$$

where $n$ is the number of the order parameter components, i. e., we have a system of $2 n$ equations which in the general case can be hardly solved. Even if we do a numerical calculation, it will be very difficult to get physically reasonable information from the obtained data.

The initial microscopic model, Eq. (1), is degenerate with respect to the rotation of all the spins through one and the same angle and this kind of degeneracy is preserved in the Landau energy. It can be partially lifted, if we take into account the effects like the spin-orbit interaction or the presence of an external magnetic field. In the exchange approximation we can find only the magnitudes of the order parameter vectors and the angles between them, namely, $\cos \left(\widehat{\varphi_{1} \varphi_{2}}\right)$. 


\section{V. SHOPOVA, T. L. BOYADJIEV}

It is well established that in a very big class of magnetic substances the exchange interaction is several orders bigger than all other magnetic interactions present in the system under consideration, so they are mainly responsible for the setting of the magnetic order (for a thorough discussion of this statement, see [6]). Having in mind these arguments we shall try to simplify the system of equations (12). For this purpose we introduce the following notations

$$
\begin{array}{ll}
\varphi_{1 i} & =\varphi_{1} \gamma_{i}, \quad \varphi_{2 i}=\varphi_{2} \alpha_{i} \\
\varphi_{1} & =\left(\sum_{i=1}^{n} \varphi_{1 i}^{2}\right)^{1 / 2}, \quad \varphi_{2}=\left(\sum_{i=1}^{n} \varphi_{2 i}^{2}\right)^{1 / 2}
\end{array}
$$

where the direction cosines $\alpha_{i}$ and $\gamma_{i}$ obey the conditions

$$
\sum_{i=1}^{n} \alpha_{i}^{2}=1, \quad \sum_{i=1}^{n} \gamma_{i}^{2}=1
$$

We shall substitute these new variables in the expression for the Landau energy, Eq. (9), and find its extrema under condition (14). The resulting system of equations has the form:

$$
\begin{aligned}
& \frac{\partial g}{\partial \varphi_{1}}=\left(\tau_{1}+g_{1} \varphi_{1}^{2}+v \varphi_{2}^{2}\right) \varphi_{2}+2 v \varphi_{1} \varphi_{2}^{2}\left(\sum_{i=1}^{n} \alpha_{i} \gamma_{i}\right)^{2} \\
& +\varphi_{2}\left(3 w_{1} \varphi_{1}^{2}+w_{2} \varphi_{2}^{2}\right)\left(\sum_{i=1}^{n} \alpha_{i} \gamma_{i}\right)=0 \\
& \frac{\partial g}{\partial \varphi_{2}}=\left(\tau_{2}+g_{2} \varphi_{2}^{2}+v \varphi_{1}^{2}\right) \varphi_{1}+2 v \varphi_{2} \varphi_{1}^{2}\left(\sum_{i=1}^{n} \alpha_{i} \gamma_{i}\right)^{2} \\
& +\varphi_{1}\left(w_{1} \varphi_{1}^{2}+3 w_{2} \varphi_{2}^{2}\right)\left(\sum_{i=1}^{n} \alpha_{i} \gamma_{i}\right)=0 \\
& \frac{\partial g}{\partial \gamma_{i}}=\left(\tau_{1}+g_{1} \varphi_{1}^{2}+v \varphi_{2}^{2}\right) \varphi_{1} \gamma_{i}+2 v \varphi_{1} \varphi_{2}^{2} \alpha_{i}\left(\sum_{i=1}^{n} \alpha_{i} \gamma_{i}\right) \\
& +\varphi_{2}\left(w_{1} \varphi_{1}^{2}+w_{2} \varphi_{2}^{2}\right) \alpha_{i}+2 w_{1} \varphi_{1}^{2} \varphi_{2} \gamma_{i}\left(\sum_{i=1}^{n} \alpha_{i} \gamma_{i}\right)=0 \\
& +\varphi_{1}\left(w_{1} \varphi_{1}^{2}+w_{2} \varphi_{2}^{2}\right) \gamma_{i}+2 w_{2} \varphi_{2}^{2} \varphi_{1} \alpha_{i}\left(\sum_{i=1}^{n} \alpha_{i} \gamma_{i}\right)=0 \\
& \frac{\partial g}{\partial \alpha_{i}}=\left(\tau_{2}+g_{2} \varphi_{2}^{2}+v \varphi_{1}^{2}\right) \varphi_{1} \alpha_{i}+2 v \varphi_{2} \varphi_{1}^{2} \gamma_{i}\left(\sum_{i=1}^{n} \alpha_{i} \gamma_{i}\right)
\end{aligned}
$$

and looks again too formidable for an appropriate analysis.

The above stated physical arguments give the oppor- tunity to simplify the problem, because we have to find only the quantities $\varphi_{1}, \varphi_{2}$ and $\sum_{i}^{n} \alpha_{i} \gamma_{i}=\cos \left(\widehat{\boldsymbol{\varphi}_{1} \boldsymbol{\varphi}_{2}}\right)$. As a first step we can determine the mutual orientation of the vector order parameters. There are three possible solutions for the quantity $\sum_{i}^{n} \alpha_{i} \gamma_{i}$. The first one is given by the relation

$$
2 v \varphi_{1} \varphi_{2} \sum_{i=1}^{n} \alpha_{i} \gamma_{i}+w_{1} \varphi_{1}^{2}+w_{2} \varphi_{2}^{2}=0
$$

and describes a general complex noncollinear magnetic phase.

The other two solutions look as follows:

$$
\sum_{i=1}^{n} \alpha_{i} \gamma_{i}= \pm 1
$$

the solution with the minus sign when substituted in Eqs. (15) gives a system of equations for $\varphi_{1}$ and $\varphi_{2}$ which has no solution from the mathematical point of view.

In the remaining part we shall discuss only the plus solution, Eq. (17) which gives a parallel orientation of vector order parameters. In order to identify properly the ordered phases we have to point out how the vector order parameters $\boldsymbol{\varphi}_{1,2}$ can be connected with the physical quantities, such as average sublattice magnetizations $\mathbf{m}_{1,2}$. All other important quantities like total magnetization $\mathbf{M}$ and staggered magnetization $\mathbf{L}$, can be expressed through them in a straightforward way: $\mathbf{M}=\mathbf{m}_{1}+\mathbf{m}_{2}$ and $\mathbf{L}=\mathbf{m}_{1}-\mathbf{m}_{2}$. The derivation of the relations between the average sublattice magnetizations and the order parameters $\boldsymbol{\varphi}_{1}$ and $\boldsymbol{\varphi}_{2}$ is straightforward because of the symmetry correspondence between the initial spin vectors and the introduced by HST vector fields (see the remark after Eq. (3)):

$$
\begin{aligned}
& \mathbf{m}_{1}=\frac{S_{0}}{\sqrt{\lambda_{1}}} \boldsymbol{\varphi}_{1}-\frac{S_{1}}{\sqrt{\lambda_{2}}} \boldsymbol{\varphi}_{2}, \\
& \mathbf{m}_{2}=\frac{S_{1}}{\sqrt{\lambda_{1}}} \boldsymbol{\varphi}_{1}-\frac{S_{0}}{\sqrt{\lambda_{2}}} \boldsymbol{\varphi}_{2},
\end{aligned}
$$

where $S_{1,0}$ are defined by Eqs. (5) for $\mathbf{k}=0$.

It is obvious from Eqs. (18) that for parallel $\boldsymbol{\varphi}_{1}$ and $\boldsymbol{\varphi}_{2}$, the sublattice magnetizations will be collinear. In this case we can simplify the mean-field equations by introducing new variables through the transformation:

$$
\begin{aligned}
& \boldsymbol{\xi}=S_{0} \sqrt{\lambda_{1}} \varphi_{1}-S_{1} \sqrt{\lambda_{2}} \varphi_{2}, \\
& \boldsymbol{\eta}=S_{1} \sqrt{\lambda_{1}} \varphi_{1}+S_{0} \sqrt{\lambda_{2}} \varphi_{2} .
\end{aligned}
$$

The Landau energy, Eq. (9), has a more simple form after expressing it with the help of the above introduced variables (19): 


$$
g=\frac{a_{1}}{2} \eta^{2}+\frac{a_{2}}{2} \xi^{2}-a \xi \eta+\frac{u}{4}\left(\xi^{4}+\eta^{4}\right) .
$$

Here the quantites $\xi=|\boldsymbol{\xi}|$ and $\eta=|\boldsymbol{\eta}|$ are positive numbers and are the magnitudes of the respective vectors. In writing expression (20) we have taken into account that $\cos \widehat{(\boldsymbol{\xi} \boldsymbol{\eta})}=\sum_{i}^{n} \alpha_{i} \gamma_{i}=1$.

The coefficients in Eq. (20) are connected with the averaged over nearest neighbours microscopic exchange parameters in the following way:

$$
\begin{aligned}
& a_{1,2}=\frac{J_{1,2}}{D}-\frac{1}{n}, \\
& a=\frac{K}{D}, \\
& D=J_{1} J_{2}-K^{2}>0 .
\end{aligned}
$$

The mean field equations are obtained directly as first derivatives of Eq. (20) for the Landau energy with respect to $\xi$ and $\eta$ :

$$
\begin{aligned}
& a_{1} \eta-a \xi+u \eta^{3}=0, \\
& a_{2} \xi-a \eta+u \xi^{3}=0,
\end{aligned}
$$

where $u$ is given by Eq. (11). It can be checked that the same system of equations will be obtained, if we minimize the Landau energy expressed by the vectors $\boldsymbol{\xi}$ and $\boldsymbol{\eta}$ under the additional condition $\sum_{i}^{n} \alpha_{i} \gamma_{i}=1$. System (22) coincides in form with the mean field equations for the Ising model [4] but is valid for the general form of the Landau energy, Eq. (9), only for a parallel orientation of $\varphi_{1}$ and $\varphi_{1}$.

\section{RESULTS AND DISCUSSION}

We have to point out that up to now the temperature does not enter explicitly in Eqs. (22), see also Eq. (2). This is not adequate for the numerical analysis, so we shall again introduce the explicit dependence on the temperature $T$, i. e., the exchange parameters will be again temperature dependent and respectively also the coefficients in the quadratic part of the Landau energy Eq. (20) will become temperature dependent; note that the temperature dependent exchange parameters are denoted by calligraphic letters. We shall not do the numerical calculations for a concrete magnetic substance; that is why we introduce a dimensionless temperature by the relation:

$$
x=\frac{T}{\mathcal{J}_{1}+\mathcal{J}_{2}} .
$$

We can fix the value of $\mathcal{J}_{1}$ and work with the following dimensionless parameters:

$$
\begin{aligned}
\beta & =\frac{\mathcal{K}}{\mathcal{J}_{1}+\mathcal{J}_{2}}, \\
\alpha & =\frac{\mathcal{J}_{1}-\mathcal{J}_{2}}{\mathcal{J}_{1}+\mathcal{J}_{2}} .
\end{aligned}
$$

The weak-coupling limit, i.e., the condition $\left(\mathcal{J}_{1} \mathcal{J}_{2}-\right.$ $\left.\mathcal{K}^{2}\right)>0$ becomes:

$$
\alpha^{2}+\beta^{2}<1
$$

As far as the system is symmetric with respect to the interchange of the two sublattices we may accept without a loss of generality that $\mathcal{J}_{1}>\mathcal{J}_{2}$.

The mean-field Eqs. (22) are solved by standard Newton iteration procedure and the solutions are substituted in the stability conditions:

$$
a_{1}>0, \quad\left(a_{1}+3 u \eta^{2}\right)\left(a_{2}+3 u \xi^{2}\right)-a^{2} \geq 0,
$$

in order to check the kind of extrema. The critical temperature for the ordered phase is found from the equality in the second relation of system (26). The calculations show that there exist one stable and generally two metastable phases, which may become unstable for some values of the parameters $\alpha, \beta$ and the number $n$ of the order parameter components. Our results will be illustrated by the temperature behaviour of $M$ and $L$ that is why we shall write down the connection of the variables $\boldsymbol{\xi}$ and $\boldsymbol{\eta}$ with the magnetization and the staggared magnetization:

$$
\begin{aligned}
\mathbf{M} & =\frac{1}{D}\left[\left(J_{1}-K\right) \boldsymbol{\eta}+\left(J_{2}-K\right) \boldsymbol{\xi}\right] \\
\mathbf{L} & =\frac{1}{D}\left[\left(J_{1}+K\right) \boldsymbol{\eta}-\left(J_{2}+K\right) \boldsymbol{\xi}\right]
\end{aligned}
$$

There are some controversial findings in the literature for the order of the phase transition to the stable phase. The calculations in $[4,5]$ are done analytically by some kind of expansion around the symmetric solutions, i.e when $J_{1}=J_{2}$, with the help of adequately chosen small parameters. Our numerical results show that the phase transition to the stable phase is of second order and the obtained ordered phase has a ferrimagnetic character, in sense, that both the magnetization $M$ and the staggered magnetization $L$ are different from zero. We illustrate the above statement by the temperature dependence of magnetization and staggered magnetization of the stable phase for $n=1,2,3$ given in Figs. 1, 2 and 3, respectively. All three figures show that there is no principal difference in the behaviour of $M$ and $L$ with the change of the number of order parameter components. But with the increase of the intersublattice interaction $\mathcal{K} \sim \beta, M$ and $L$ are getting closer in magnitude. For small sublattice coupling and $n \geq 2, L$ gains negative values for temperatures $x<x_{0}$. The sublattice magnetizations $m_{1}$ and 


\section{V. SHOPOVA, T. L. BOYADJIEV}

$m_{2}$ which near the critical temperature have the relation $m_{1}>m_{2}$, change in such a way with the temperature, that for $x_{0}, L=0$,i. e., $m_{1}=m_{2}$. We shall call $x_{0}$ a "compensation point" in analogy with ferrimagnetism [7] and it exists for relatively small values of the parameter $\beta$ and arbitrary values of $\alpha<1$. For the temperatures $x<x_{0}, m_{1}<m_{2}$, see Fig. $2,(\beta=0.1)$. The order of the phase transition to the stable phase is checked also by a numerical differentiation of the Landau energy for the stable phase in the critical point and a zero jump of the entropy is found.

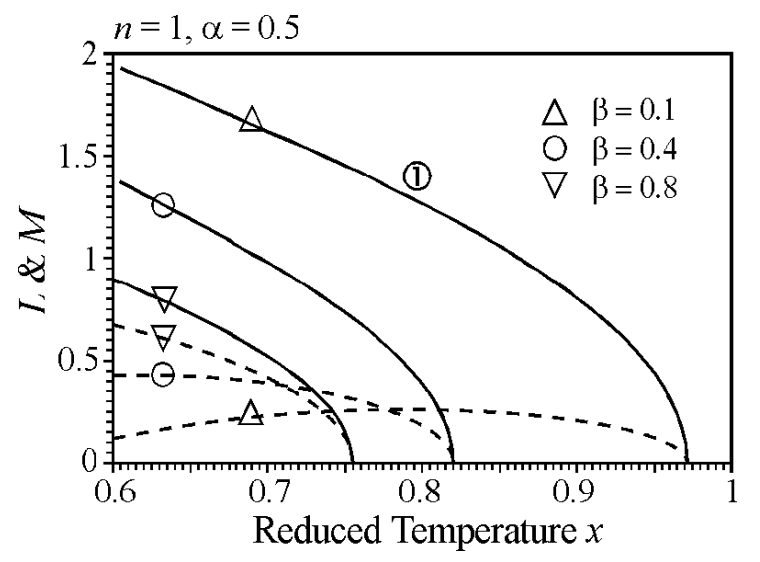

Fig. 1. The temperature dependence of $M$ (thick line) and $L$ (dashed line) of the stable phase for $n=1, \alpha=0.5$ and different values of the parameter $\beta$.

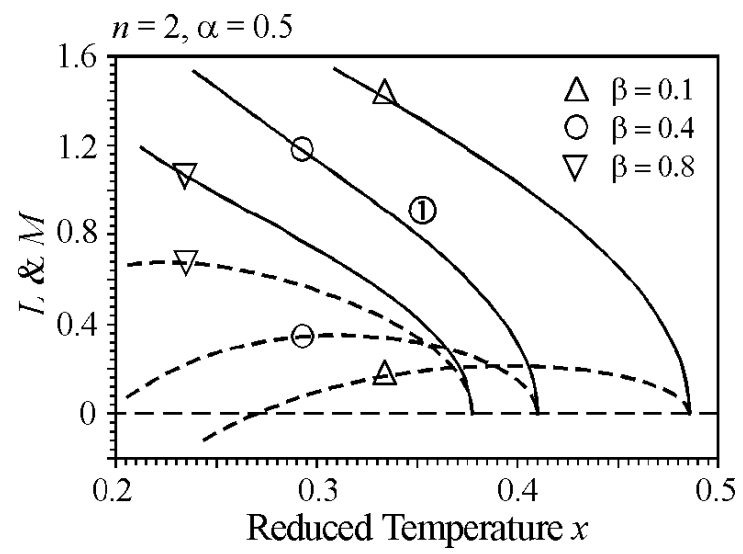

Fig. 2. The temperature dependence of $M$ (thick line) and $L$ (dashed line) of the stable phase for $n=2, \alpha=0.5$ and different values of the parameter $\beta$.

We found as mathematical solutions of the system of equations (22) two more phases. Our analysis shows that for $n=1$ these solutions are absolutely unstable. For $n \geq 2$ there exist 3 phases - two metastable phases and a stable one. First of all we shall identify the nature of the metastable phases with the help of Fig. 4, where the temperature dependence of $M$ and $L$ are given for $n=2$. For the stable phase $M>|L|$, as expected, for the whole range of temperatures. There is a "compensation point" for these concrete values of the parameters $\alpha, \beta$. The phase transition is of second order; this is also seen from Fig. 5, where the temperature dependence of the Landau energies for the three phases from Fig. 4 are given. The high-temperature metastable phase, see Figs. 4, 5, occurs through a second order phase transition and has $|L|>M$ for the whole range of parameters, for which it exists.

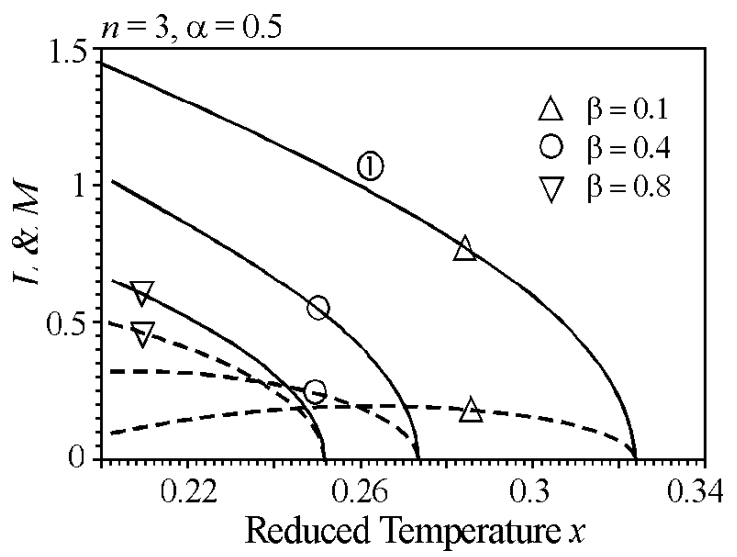

Fig. 3. The temperature dependence of $M$ (thick line) and $L$ (dashed line) of the stable phase for $n=3, \alpha=0.5$ and different values of the parameter $\beta$.

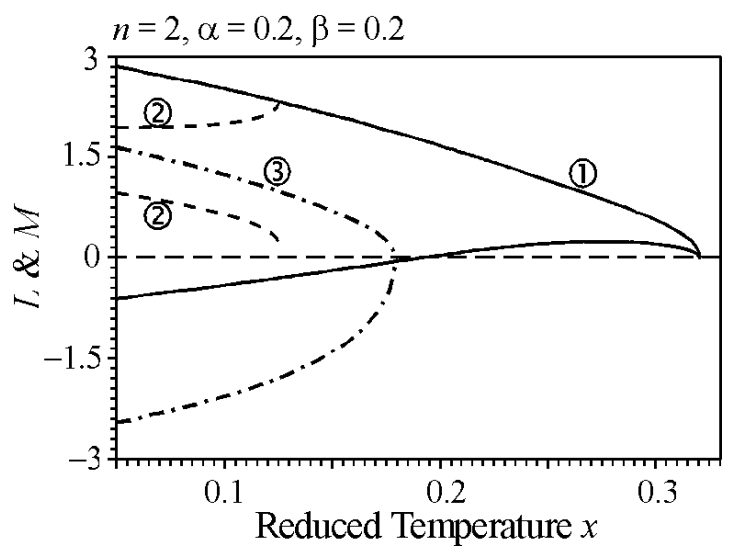

Fig. 4. The temperature dependence of $M$ and $L$ for the stable (thick line), high-temperature metastable (dash-dotted line) and low-temperature metastable (dashed line) phases for $n=2, \quad \alpha=0.2, \quad \beta=2$.

The staggered magnetization is negative, so nevertheless $\mathcal{J}_{1}>\mathcal{J}_{2}$ the sublattice magnetizations obey the relation $m_{1}<m_{2}$. The low temperature metastable phase (dashed line in Figs. 4 and 5 ) is even more exotic. It has positive staggered magnetization and again $L>M$. The phase transition to the low-temperature phase is definitely of first order. The relation between the energies of the three phases can be seen in Fig. 5. The two metastable phases described above are "inverse" because for positive coupling between the sublattices, the 
staggered magnetization is bigger in magnitude than the magnetization. It is a question of additional investigation whether the account of crystal anisotropy, dipole interaction or finite size effects may change the stability of the metastable phases.

The same picture is valid for $n=3$ and the difference between the detailed picture, given above for $n=2$, is only quantitative. The increase of the magnitude of intersublattice interaction shifts the domain of existence of the metastable phases to lower temperatures. This is well illustrated in Fig. 6, where the Landau energy of the metastable phases for two values of the parameter $\beta$ are given.

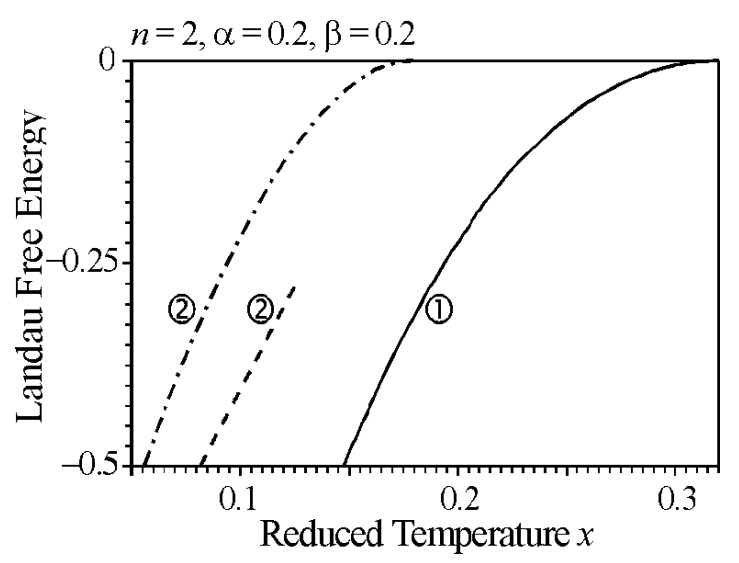

Fig. 5. The temperature dependence of Landau free energy for the stable (thick line), high-temperature metastable (dash-dotted line) and low-temperature metastable (dashed line) phases for $n=2, \quad \alpha=0.2, \quad \beta=0.2$.

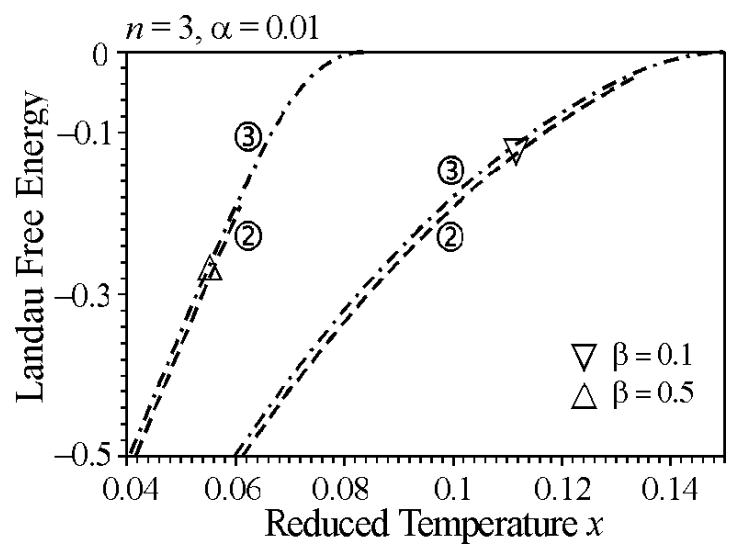

Fig. 6. The temperature dependence of Landau free energy for the high-temperature (dash-dotted line) and low-temperature (dashed line) metastable phases for $n=3$, $\alpha=0.0 .1$, and two values of $\beta$.

We have calculated the dependence of the transition temperatures of the stable $\left(x_{c}\right)$, high-temperature $\left(x_{h}\right)$ and low-temperature $\left(x_{l}\right)$ phases on the magnitude of the intersublattice exchange. This is shown in Fig. 7 for two values of the order parameter components: $n=2,3$.
With the increase of $\beta, x_{h}$ (dotted-dashed line) and $x_{l}$ (dashed line) are shifted to lower values and for some value of $\beta$ at first the low-temperature phase ceases to exist as a physical solution; the same happens with the high-temperature phase but for a bigger value of $\beta$. The point $\beta=0$ is a limiting case of fully decoupled ferromagnetically ordered sublattices.

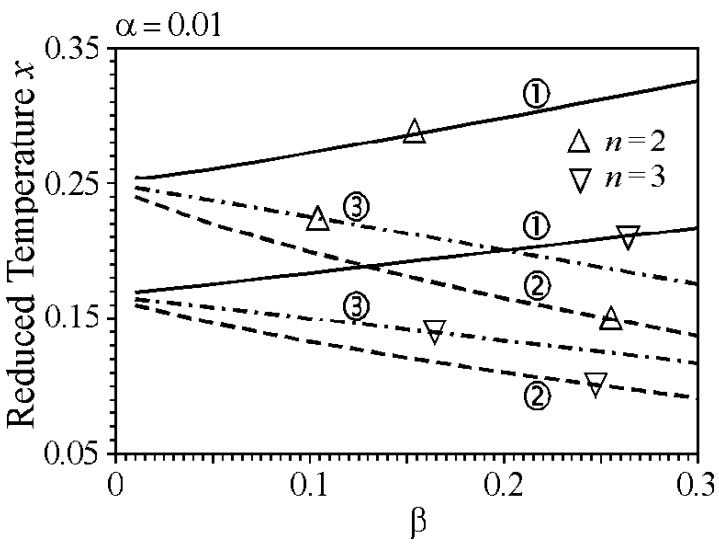

Fig. 7. The dependence of the transition temperatures of the stable (thick line), high-temperature (dash-dotted line) and low-temperature (dashed line) phases on the sublattice coupling $\beta$ for $n=2$ and $n=3$.

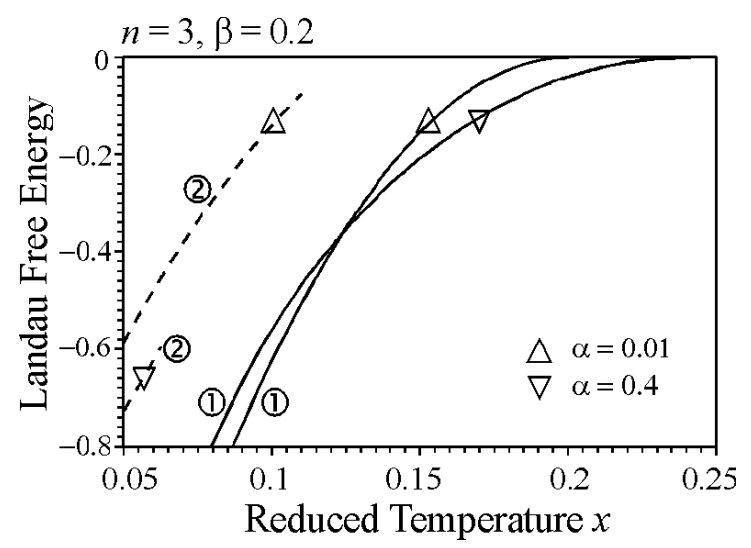

Fig. 8. The temperature dependence of Landau free energy for the stable (thick line) and low-temperature metastable (dashed line) phases for $n=3, \beta=0.2$ and two values of $\alpha$.

The same effect occurs when the difference between two sublattices grows, which is measured by the increase of the parameter $\alpha$. This is illustrated in Fig. 8, where the Landau energy of the stable and low-temperature metastable phases are given for two values of $\alpha$. It can be seen that the difference between the transition temperatures of the stable phase $\left(T_{c}\right)$ and the low-temperature phase $\left(T_{l}\right)$ becomes bigger with the increase of $\alpha$. So we can state that for big interlattice interaction and/or big difference between the sublattices the metastable phases do not exist and only the stable one is present.

In conclusion, we have analyzed one particular case, namely a positive sublattice interaction in the weak cou- 
pling limit. We suppose that for an antiferromagnetic (negative) coupling the picture will not be changed substantially, nevetheless this case needs a separate investigation. Even in the simplest case, we have presented, the model of two coupled Heisenberg subsystems exhibits quite a complex behaviour.

\section{ACKNOWLEDGEMENTS}

The authors thank Dimo I. Uzunov for valuable discussions.
[1] Yu. M. Ivanchenko, A. A. Lisyanskii, A. E. Fillipov, Fluctuazionnie effecti $v$ sistemah s konkurirujustimi vzaimodeistvijami (Naukova Dumka, Kiev, 1989); in Russian.

[2] R. Brout, Phys. Rep. 10, 1 (1974).

[3] D. I. Uzunov, Introduction to the Theory of Critical Phenomena (World Scientific, Singapore, 1993); D. I. Uzunov, in Lecturess on Cooperative Phenomena in Condensed Matter, edited by D. I. Uzunov (Heron Press, Sofia, 1996), p. 46.

[4] Hasan M. Al Mukadam, D. I. Uzunov, Physica A 232, 326 (1996).

[5] C. Oleksy, J. Przystawa, Physica A 121, 145 (1983).

[6] Yu. A. Izyumov, B. E. Naish, S. B. Petrov, J. Magn. Magn. Mater. 13, 275 (1979).

[7] S. V. Vonsovskii, Magnetism (Magnietizm) (Nauka, Moscow, 1971).

\title{
АНАЛІЗ МЕТОДОМ СЕРЕДНЬОГО ПОЛЯ ДВОХ ЗВ'ЯЗАНИХ МОДЕЛЕЙ ГАЙЗЕНБЕРІА
}

\author{
Д. В. ШШопова ${ }^{1}$, Т. Л. Бояджев ${ }^{2}$ \\ ${ }^{1}$ Лабораторія СРСМ, Інститут фізики тьердого тіла Г. Наджакова, \\ Болгарсъка академіл наук, $B G-1784$, Болгаріл \\ ${ }^{2}$ Факультет математики $і$ комп'ютерних наук \\ Софійсъкий університет, $B G-1164$, Боларія
}

Отримано і проаналізовано вираз для вільної енертії Ландау двох зв'язаних гайзенбергівських підсистем у загальному випадку $n$-компонентних параметрів порядку. Обчислення проведено в гранипі слабкого зв'язку, коли обмінні взаємодії $J_{1}$ та $J_{2}$ в підсистемах перевищують міжпідсистемну взаємодію $K$. Залежно від знака $K$ існують дві стійкі колінеарні фази. Фазові переходи до стійких фаз є фазовими переходами другого роду для всього набору розглянутих параметрів моделі. Чисельні розрахунки показують, що для додатного знака взаємодії існують ще дві фази, які $є$ метастабільними або нестійкими залежно від кількости компонент $n$ параметра поря,Дку. 